\title{
Brûler, casser, détruire, se réjouir.
}

Contribution à l'étude du vocabulaire des funérailles chez les Goun (Bénin)

Burning, Breaking, Destroying, Rejoicing: Contribution to a Study of Funeral Vocabulary among the Gun (Benin)

\section{Gilbert Rouget}

\section{(2) OpenEdition}

\section{Journals}

Édition électronique

URL : http://journals.openedition.org/span/1354

DOI : $10.4000 /$ span. 1354

ISSN : 2268-1558

Éditeur

École pratique des hautes études. Sciences humaines

Édition imprimée

Date de publication : 1 décembre 1994

Pagination : $9-41$

ISSN : 0294-7080

\section{Référence électronique}

Gilbert Rouget, «Brûler, casser, détruire, se réjouir. 》, Systèmes de pensée en Afrique noire [En ligne],

13 | 1994, mis en ligne le 16 février 2014, consulté le 30 avril 2019. URL : http://

journals.openedition.org/span/1354; DOI : 10.4000/span.1354 


\section{BRÊLER, CASSER, DÉTRUIRE, SE RÉJOUIR. Contribution à l'étude du vocabulaire des funérailles chez les Goun (Bénin) $)^{1}$}

par

Gilbert Rouget

Chez les Goun comme chez tant d'autres peuples le rituel funéraire fait abondamment appel, sous des formes très diverses, à la musique. Ayant à décrire, dans le cadre d'un ouvrage en préparation sur la musique de cour à Porto-Novo², celle qui est liée au rituel funéraire du roi, je me suis trouvé dans l'obligation de préciser le sens de certains termes concernant le rituel. L'un d'eux, ag $\mathfrak{\jmath}$, qui entre dans la composition d'un mot désignant un certain type de musique funéraire, pose comme on va le voir bien des problemes. On ne peut espérer les résoudre, semble-t-il, qu'en examinant les différents usages de ce terme, en les mettant en relation avec le plus possible d'idées et de pratiques sous-tendant la logique de ce rituel funéraire et, pour dégager cette logique, en comparant avec ce qui s'observe chez les

${ }^{1}$ Michel Cartry a bien voulu me faire part de certaines de ses réflexions sur ce texte, avant de l'envoyer à l'impression. Qu'il en soit ici vivement remercié. Ses remarques m'auront permis d'ajouter sur quelques points d'utiles précisions et de dissiper ainsi certaines ambiguïtés.

2 Un bref descriptif en a récemment été publié (Rouget, 1991).

Le deuil et ses rites III

Systèmes de pensée en Afrique noire, 13, 1994 
voisins des Goun. C'est ce qui va être tenté ici. Mais avant d'en venir au mot lui-même et à ce qu'il désigne, il importe de donner au lecteur un aperçu du déroulement général de ce rituel.

Traditionnellement celui-ci s'échelonnait - et s'échelonne souvent encore, de nos jours - suivant quatre grandes étapes. Dans l'ordre: 1) l'inhumation du (de la) mort(e) (m€kúkú), peu après le décès, en général la nuit suivante, suivie, quelques jours après, de la crémation de certaines affaires (ag $\zeta$ vfvé mime) du (de la) disparu(e); 2) plusieurs mois après - neuf pour un homme, en principe, et sept pour une femme, mais souvent bien plus - l'exhumation du crâne (ayísún diqe), toiletté, puis mis en quelque sorte en réserve; 3) dans un délai parfois très bref (pour le roi), mais parfois très long (pour le commun), l'exposition et la vénération du crâne (aytsún giglo) puis l'enterrement définitif et secret de celui-ci (ayísún dó ohún) ${ }^{3}$, suivi de l'appel du trépassant ${ }^{4}$ (cĺys wíws), d'une nouvelle cérémonie de crémation (ags mime $q a x \zeta$ ) et de plusieurs jours de grandes réjouissances (ag $\ q u q u$ ); 4) ici encore dans des délais très variables suivant les cas, la mise en place d'un autel commémoratif (asányi qó te) du (de la) disparu(e), désormais promu(e) défunt(e) (kúviţ $)^{5}$.

Ces quatre grandes étapes, formant en fait quatre suites différentes de rituels partiels, séparées par des temps de longueurs très variables, peuvent être vues comme divisant le rituel funéraire général en deux phases successives qui pourraient être dites, la première "mortuaire", la seconde "consécrationnelle", la phase mortuaire commençant par l'inhumation du mort et finissant par l'exhumation de son crâne, la phase consécrationnelle débutant par la vénération du crâne et s'achevant par l'installation de l'autel commémoratif du défunt. Cette division en deux phases reste, sauf erreur, valable quelles que soient les variantes affectant, d'un sous-groupe ethnique à l'autre, la succession

${ }^{3}$ Litt.: "le crâne prend la pirogue". On dit aussi, au lieu de ayísún, cíys, "le trépassant" prend la pirogue. Une cérémonie de ctyo dó hún a été longuement décrite chez les Fon par B. Adoukonou, mais dans le cas à vrai dire très particulier de défunts "morts en forêt, ( $\ldots)$ à la guerre, (..) noyés, (..) en voyage", bref "hors de l'enclos familial" (Adoukonou, 1979: 143 et 144). Nous y reviendrons plus loin, à la note 13 .

$4 \mathrm{Cf}$. plus loin note 12 .

$5 \mathrm{Cf}$. plus loin note 16 . 
et la composition des quatre grandes étapes décrites ci-dessus. Pour désigner la seconde de ces phases, dite à l'instant "consécrationnelle", les Goun emploient souvent, lorsqu'ils s'expriment en français, le terme "funérailles". C'est précisément dans ce sens qu'il figure en tête de cette étude.

La forme sous laquelle les pages qui suivent se présentent - celle d'un article de dictionnaire - tient à ce qu'il s'agit d'un texte conçu, primitivement au moins, pour venir en annexe de l'ouvrage dont on vient de parler et pour y prendre place parmi d'autres entrées concernant d'autres termes du vocabulaire. Celles-ci ne figurant pas ici, le sens de quelques-uns de ces termes a été indiqué en note.

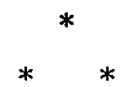

$A g$ ร. $1^{\circ}$ Simulacre d'abri que l'on brûle au cours du rituel funéraire. $2^{\circ}$ Collection d'objets ayant appartenu au défunt, que l'on brûle en même temps. $3^{\circ}$ Rituel de crémation ${ }^{6}$ de ces différents objets. $4^{\circ}$ Réjouissances constituant la suite obligée de ce rite. $5^{\circ}$ Grandes réjouissances collectives. - L'ordre suivi pour indiquer ces différentes acceptions du mot montre assez comment celui-ci a évolué au cours des temps: métonymies successives et élargissement du sens se sont conjugués pour le faire passer du concret à l'abstrait et du spécifique à l'indéterminé. Le démontrer requerra toutefois, allons-nous voir, d'assez longs développements.

Le mot agS apparaît pour la première fois dans la littérature, semble-t-il, en 1875 , sous la plume du R. P. Baudin. Décrivant, dans une lettre datée du 10 avril de cette année, les "funérailles des quatre derniers rois de Porto-Novo", qui venaient d'avoir lieu, il fait emploi

${ }^{6}$ Bien que "crémation" soit ici un peu détourné de son sens habituel puisqu'il s'agit de mettre au feu et de brûler non point "les corps des défunts" (Littré), mais des objets leur ayant appartenu - action bien différente -, ce mot a finalement paru préférable à celui, purement technique, de "brûlage", en raison de ses relations avec le rituel funéraire. Ce choix ne signifie en rien, bien entendu, qu'on ait voulu réduire l'une à l'autre la symbolique des deux opérations. 
du mot à sept reprises et l'utilise pour commencer - ce qui est significatif, allons-nous voir - en association avec le mot nago (ou yorouba) sba, "roi"7.

"Dès la veille de la neuvaine, sur le soir, une cabane de bambous, recouverte de paille, est disposée [au palais] pour recevoir l'ago oba, c'est-à-dire la collection d'objets que l'on veut envoyer aux rois défunts", écrit-il (Baudin 1875: 615). Dans l'emploi suivant (ibid.: 616) qu'il fait de "ago", le mot est traduit par "case" et désigne cette fois, par conséquent, non plus la "collection d'objets" destinés aux rois defunts, mais cette "cabane de bambous recouverte de paille" construite pour les recevoir. Un peu plus loin toutefois, ce sont à nouveau les objets que le mot désigne: "Le neuvième jour, écrit Baudin (ibid.: 629), (...) on se prépare à brûler et à expédier les présents aux rois défunts". La procession se dirige "hors des remparts de la ville", "au pied d'un grand arbre où l'on doit brûler l'ago", dont les "objets", précise-t-il, sont portés par les femmes.

Un demi-siècle plus tard, en 1929, le mot ags apparaît dans l'étude que publie l'abbé Kiti, sur les "Rites funéraires usités chez les Alladanou (...)". Les "Cérémonies d'ago" (Kiti, rééd. 1968: 17) - le mot désignant cette fois non plus des objets concrets mais des rituels y sont données pour des "réjouissances en l'honneur des défunts". Au terme de diverses cérémonies étalées sur plusieurs jours et marquées par de grandes ripailles, les femmes, écrit-il (ibid.: 19) "se mettent à balayer l'interieur des maisons (...)", puis "entassent les ordures" dans un kplakpla [natte en molle de palmier-raphia], après quoi celles-ci sont portées en "brousse, en un lieu appelé agotô [lisez agł to], où elles sont brûlées en présence de la famille (...)". Il s'agit donc ici, non plus de "présents" expédiés en les brûlant aux défunts, comme le

7 Dans les pages qui suivent on a respecté, dans les citations, l'orthographe de l'auteur cité, à ceci près toutefois: 1) qu'en goun la voyelle nasale sera indiquée, conformément aux recommandations de la Commission nationale de linguistique de la République du Bénin, par l'ajout de $n$ à la voyelle orale correspondante; 2) qu'en yorouba on trouvera $o$ au lieu de $o$.

Sur la phonologie de la langue goun, voir Rouget 1972, sur la tonétique, Rouget 1974. 
rapporte Baudin, mais d'"ordures". Kiti n'en ajoute pas moins, aussitôt: "Certains membres de la famille prennent alors qui un très beau pagne, qui un chapeau (...), celui-là des flacons de parfum, etc... et dit 'Je brûle ceci en l'honneur de mon père (...) défunt'. On admire son geste (...)". Mais, comme on voit, ici encore Kiti s'écarte de Baudin: le but de l'operation n'est plus "d'expédier des présents" aux défunts (nous reviendrons plus tard sur ce point), mais de leur faire "honneur".

Quinze ans après, en 1953, Akindélé et Aguessy publient à leur tour, dans leur célebre ouvrage (1953: 108-110), une courte description de la "cérémonie" de "ago", présentée cette fois comme étant la "rupture des dernières attaches naturelles [du défunt] avec le monde présent". Certes il s'agit, ici encore, de brûler cérémoniellement des objets, mais en vue ni de "faire honneur" au mort, ni de lui "expédier des présents". Si l'on va "brûler l'ago", expliquent-ils, c'est parce que:

"certains effets du défunt sont considérés comme son enveloppe.

L'esprit étant parti, l'enveloppe du corps ne saurait demeurer en ce monde. Quiconque en userait ne pourrait vivre en paix, car il ne pourrait empêcher l'esprit du propriétaire de visiter ses effets."

Ceux-ci sont donc "livrés aux flammes, sur une petite place aménagée dans la forêt (...). Tam-Tam et chants accompagnent le cortège".

Ago, avaient noté, pour commencer, ces deux auteurs, est un mot "tiré du yorouba" qui signifie "couverture ou enveloppe du revenant", ce dernier terme étant de toute évidence, bien qu'ils n'en disent rien, celui qui désigne, à Porto-Novo comme ailleurs, en pays yorouba, les célèbres (yor.) Egúngún, masques d'une société secrète consacrée au culte des morts, connus en français sous le nom de "Revenants".

"Collection d'objets", "présents destinés aux rois défunts", "case édifiée pour abriter ces objets", "balayures", objets "de valeur", "effets" ayant appartenu au mort, "couverture ou enveloppe du Revenant", telles sont, à lire ces auteurs, les différents sens attribués au mot ago (lisez aģ) lorsqu'il désigne une chose concrète. Lorsqu'il se réfère à une action, c'est pour la dénoter sous deux aspects différents, l'un consistant à brûler des objets au cours d'un rituel funéraire, l'autre consistant, pour le groupe concerné, à se réjouir collectivement en cette occasion. Curieux vocable, en vérité ! Car s'il me faut maintenant 
dire que ces différents sens du mot agł sont bien ceux que j'ai moimême recueillis au cours de mes recherches en pays goun, il me faut surtout souligner qu'en goun il ne se rapporte en propre - je veux dire par là en dehors du contexte précis de ce rituel - à aucun des objets, ni à aucune des actions que l'on vient de nommer. Bref, pris isolément, il ne désigne, en goun, ni une "case", ni une "collection d'objets", ni un "présent", ni une "ordure", ni une "enveloppe"; de même ne sert-il à la formation d'aucun terme désignant une action telle que "brûler", "honorer", "faire don de".

Akindélé et Aguessy y voient, a-t-on dit, un mot "tiré du yorouba". Serait-ce la clé de l'énigme ? Consulté à Porto-Novo, en 1958, sur le bien-fondé de cette étymologie, un Porto-Novien nago, yoroubaphone par conséquent, mais de plus particulièrement bien informé des choses touchant aux Egúngún, aux "Revenants", en même temps que très instruit des rites funéraires des Goun, s'était récrié. Nous venions d'assister ensemble à une cérémonie de agł. Aucune ambiguïté concernant la question soulevée n'était donc à craindre; nous parlions bien de la même chose. Selon lui, le mot yorouba auquel ces deux auteurs se réferrent était ag. (ton bas) et non ags (ton haut). Par ailleurs, contrairement au ag.S des Goun, qui est destiné à être brûlé, le "pagne" (yor. ags) des "Revenants" yorouba ne l'était, disait-il, en aucun cas. Il n'y avait donc pas plus de rapport entre les choses qu'entre les mots. Cela étant, l'origine du mot agł proposée par Akindélé et Aguessy paraissait difficilement recevable. Faute d'enquête menée sur ce point, la question devait rester longtemps encore, pour moi, sans réponse.

Reprenant le problème en 1991, en vue de rédiger ces lignes, la lecture du dictionnaire yorouba d'Abraham (1958) devait m'ouvrir une nouvelle piste. A côté de ags (ton bas), "costume worn by the Egúngún", on y trouve en effet un autre mot, ag 5 , cette fois rigoureusement homophone de celui qui nous occupe et signifiant "tente" (angl. tent). Ce qu'on entend couramment par ce terme ne fait pas partie, dans cette région d'A frique, des produits traditionnels de l'artisanat. L'existence dans ce dictionnaire d'un terme y figurant avec pour seule traduction le mot "tente" avait de quoi surprendre. Une phrase en yorouba donnée à la suite, à titre d'exemple, et traduite: "make this 
mat into a tent", devait toutefois éveiller chez moi un souvenir très net, lié précisément a cette cérémonie de ag $\mathfrak{b}$ dont je viens a l'instant de parler et qu'il me faut maintenant présenter en peu de mots.

C'était en 1958, à Gbékandji, petite localité sise à quelques kilomètres au nord de Porto-Novo. Une femme du village, décédée une dizaine de jours auparavant, y avait été inhumée. La première phase de son rituel funéraire venant à son terme, les préparatifs en vue d'aller "en brousse" pour y brûler le ag $\mathfrak{g}$ de la morte étaient en cours. Pour ce faire, une natte en kplakpla pliée en deux de manière à former un toit à deux pentes fut posée sur le sol pour servir en quelque sorte d'abri à ce qui semblait être un petit amas d'offrandes déposées là, par terre. La photographie ci-apres permettra au lecteur de juger de l'effet produit. On en conviendra sans peine, je suppose, aux yeux d'un Européen il est tout à fait celui d'une "tente" en miniature. Je n'ai malheureusement pas eu, sur le moment, l'esprit de demander de quel mot on désignait, dans ce village, cette construction sommaire. Le plus courant, devais-je néanmoins apprendre par la suite, consiste à la nommer kplakpla.

En 1991, l'examen de cette photographie prise en 1958 devait tout naturellement m'apparaître comme illustrant parfaitement la phrase citée par Abraham comme exemple du mot (yor.) ags traduit (angl.) "tent". Il devenait dès lors très probable: 1) que le mot ags, qui sert chez ces villageois comme dans le reste du pays goun, à désigner à la fois les objets à brûler au cours du rituel funéraire et, plus globalement, le rituel lui-même, devait également désigner - ou avoir désigné - cette natte repliée en forme de tente, destinée elle aussi à être brûlée; 2) que ce mot était un emprunt au yoruba. D'autres données devaient rapidement consolider l'hypothèse.

En premier lieu, celles tirées d'une nouvelle lecture, éclairée par les faits précédents, de l'étude de l'abbé Kiti. Dans sa relation des "cérémonies d'ago", celui-ci parle à deux reprises de l'emploi fait à cette occasion de la natte dite kplakpla, dont il a précédemment parlé. On y ramasse, a-t-on vu, les "balayures" pour aller les brûler en brousse (Kiti 1968: 20), mais on en fait également un tout autre usage, quelques jours avant, à un autre moment du rituel. Il s'agit alors de convier les âmes des défunts à un repas disposé à leur intention par 
terre, sur "un petit tas de poussière" au-dessus duquel on étend un kplakpla "de manière à le couvrir comme d'une petite tente". Nous retrouvons donc ici, dans le contexte des "cérémonies d'ago" (Kiti, op. cit.) la natte [repliée] en forme de tente décrite par Abraham s.v. (yor.) ag $\zeta$ et photographiée, a-t-on vu, dans les environs de PortoNovo au cours d'un rituel de agj. Les choses ne sont toutefois pas aussi simples.

L'officiant, précise Kiti (ibid.: 18), appelle le défunt à venir [je cite] "manger dans l'avo (la tente élevée sur le tas de terre)". A lire ce texte, cette "tente" (en kplakpla, rappelons-le, c'est-à-dire en natte, l'auteur lui-même vient de le dire) serait donc dénommée "avo" et non "ago" [transcription de Kiti] ou [ma transcription] "ag $\zeta$ ", comme je viens d'en faire l'hypothese. Mais s'il en est ainsi, demandera-t-on, pourquoi cette hypothèse et pourquoi lire ago là où est écrit avo ? Parce qu'il s'agit, suivant moi, d'une faute d'impression. Reste toutefois à le démontrer, ce que, pour ne pas retarder le lecteur, la note 8

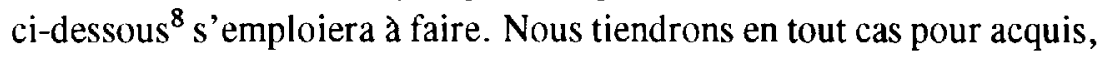

${ }^{8}$ Si dans ses transcriptions des mots goun l'abbé Kiti n'indique pas les tons, il n'en distingue pas moins soigneusement $[0]$ et $[o]$ qu'il écrit respectivement $o$ et $\hat{o}$; exemple agł to "lieu [de la crémation] de ag $\jmath^{\prime \prime}$, qu'il écrit, a-t-on vu, agotô. Le mot qui nous occupe et qu'il écrit avo ne peut donc être que avs ou avs, deux mots parfaitement attestés en goun, le premier signifiant "pagne, étoffe, vêtement" - donc "tissu" et non "natte" (kplakpla) - le second désignant, suivant le contexte, "un sacrifice de renvoi", comme l'indique excellemment Maupoil (1943: 344 note 1) mais aussi, à Porto-Novo du moins, l'esprit malfaisant auquel on le destine (sur cet avj, voir Akindélé et Aguessy, 1953: 125-127). Que ce soit avs ou avs, donner le mot comme désignant une "tente" dont il est précisé, quelques lignes plus haut, qu'clle est en kplakpla ne peut donc être qu'une erreur. Mais pas de traduction car il est hors de question que l'abbé Kiti, qui était goun et qui, ses écrits le prouvent, connaissait parfaitement ce vocabulaire, en ait fait une de ce genre. Il s'agit donc, sans aucun doute possible, d'une faute d'impression. Tout indique, en conséquence, qu'à la place de avo c'est bien en effet ago - i.e. ag $\delta$ - qu'il faut hire: aucun autre terme n'est concevable dans ce contexte. Un mot toutefois avant d'en finir sur ce point. L'étude de l'abbé Kiti sur les "Rites funéraires..." a d'abord paru en 1929 et 1930 dans la Revue des Missions catholiques de Lyon. La faute y avait-elle déjà été faite ? Je n'ai pas eu le loisir d'en faire la recherche. 


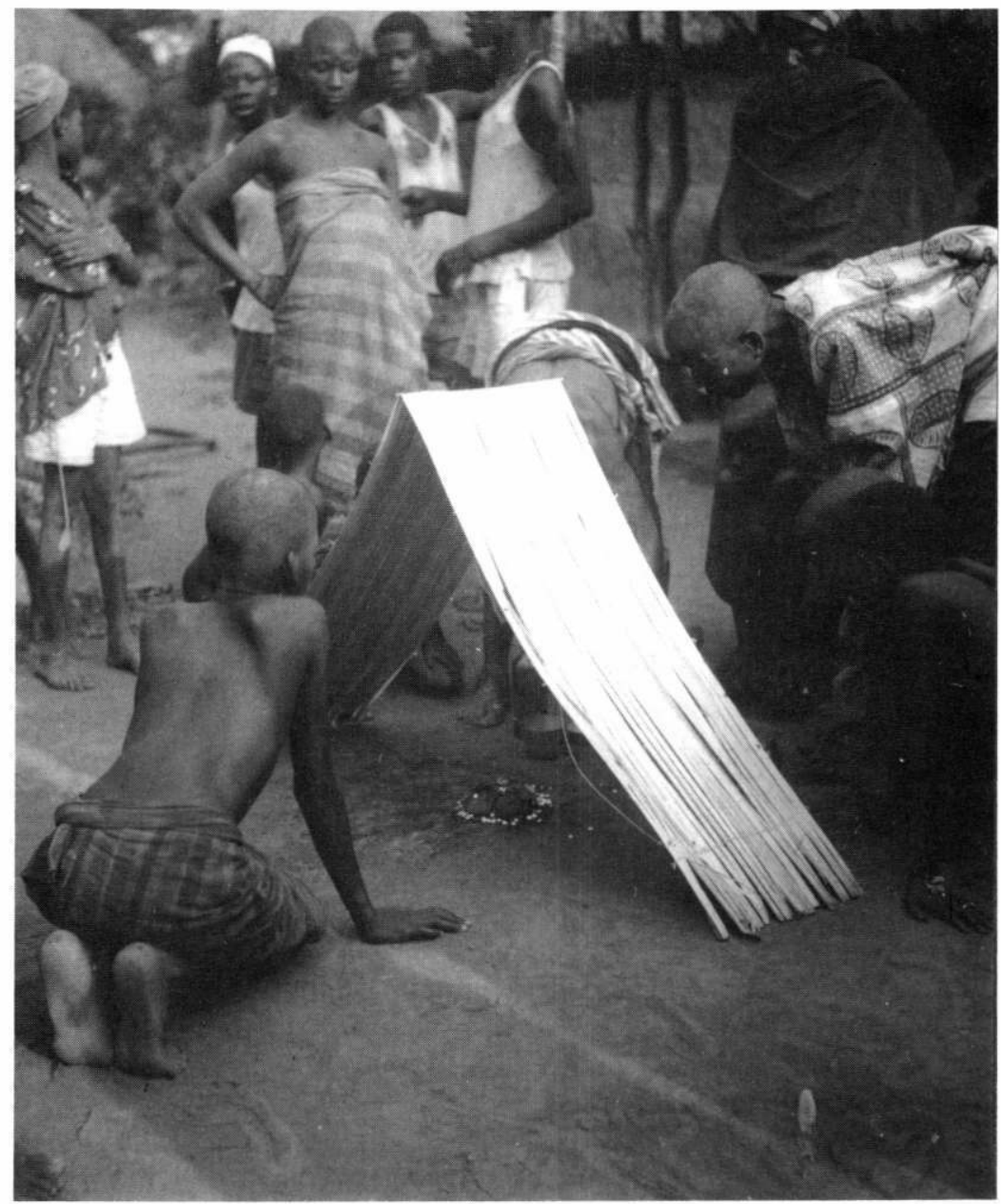

Préparatifs du aģ mim€, "crémation de aģ".

Sous la natte en kplakpla repliée (ag $f$ ?), mise en place il y a un instant, le sang d'une poule qui vient d'être égorgée est répandu sur les débris de calebasse que la fillette (à genoux au premier plan) a soigneusement disposés sur un socle en bouse de vache posé par terre, avec sur le pourtour des grains de maïs. Village de Gbékandji, 2 janvier 1959 (Photo G. Rouget). 
désormais, que le goun ags est bien, comme l'ont dit Akindélé et Aguessy, un emprunt fait au yorouba, mais que le mot emprunté n'est pas celui qu'ils indiquent, la confusion provenant d'une erreur de ton et s'expliquant par le fait que le mot yorouba invoqué, ags, "costume de Revenant", qui renvoie à toute autre chose, appartient lui aussi au vocabulaire du rituel funéraire.

Si dans son grand Dictionary of Modern Yoruba, qui est de 1958, Abraham n'indique pour ags qu'un seul sens, celui de "tent", le bien plus ancien $A$ Dictionary of the Yoruba Language, publie pour la première fois en 1913, qui le donne en premier, le fait suivre de plusieurs autres: "shed, tabernacle, pavilion, hovel, encampment". Consulté sur l'usage actuel de ce mot, Olabiyi Babalola Yai', de passage à Paris en 1991, devait m'apprendre que plutôt qu'une "tente" qui n'en traduit qu'un aspect très particulier, il désigne d'une manière générale un abri temporaire, un campement, une construction non définitive, un établissement provisoire. C'est ainsi que pour distinguer l'ancienne ville de Oyo ( $y 5$ ), capitale du royaume (ou de l'empire) yorouba, qui fut occupé comme on sait, par les Peul au début du XIXème siècle, de la nouvelle ville installée plus au sud, on nomme la première sys Ilé (Abraham: s.v. sys, "Old sys"), litt. "Oyo de la maison", et la seconde Ags sys, "Campement de Oyo" ${ }^{10}$. Le mot connote donc une notion de transitoire ou de provisoire qui est, semble-t-il, à mettre directement en rapport avec la nature des rituels funéraires auxquels, chez les Goun tout au moins, il est lié. Nous y reviendrons plus loin.

Mais avant de quitter le monde des Yorouba, observons que chez eux aussi la coutume est - ou en tout cas était - d'aller "en brousse" brûler "tout ce que le défunt utilisait chaque jour, sa pipe, la natte sur laquelle il dormait, la vaisselle dont il se servait pour manger [etc.]" (Ellis 1894: 159). Ce rituel de crémation des affaires du mort impliquait-il, à un moment ou à un autre, l'emploi d'une natte en forme de "tente" ou d'"abri" (yor. agł) ? La recherche, si elle n'est déjà faite,

${ }^{9}$ Linguiste béninois, de famille yorouba comme son nom l'indique, professeur à l'Université de Floride aux Etats-Unis.

${ }^{10}$ De ce nouvel Oyo, Abraham précise (s.v. Jy $>\mathrm{D}(1)$ ): "it is often disparagingly called $A g \jmath d \gamma y \zeta "$. $A g \Im$ comporterait donc, en yorouba, une nuance dépréciative. 
ce que j'ignore, mériterait d'être entreprise. Elle le mériterait d'autant plus que la coutume consistant à mettre en place, à un moment ou à un autre du rituel funéraire, une petite construction sommaire destinée à être peu après détruite, d'une maniere ou d'une autre, ne s'observe pas que chez les Goun, bien au contraire; son aire d'extension est, on va le voir, considérable. La variété des aspects qu'offre, suivant les régions, cette construction funéraire permettra d'en mieux comprendre le symbolisme.

Chez les Fon, le rituel funéraire se divise en deux phases successives qui pourraient être dites, elles aussi, comme j'ai proposé de le faire pour les distinguer clairement l'une de l'autre chez les Goun ${ }^{11}$, la première "mortuaire", la seconde "consécrationnelle". Cette dernière a êté décrite par Herskovits (1938, I: 194-208) au chapitre intitulé "The ancestral cult: deification of the ancestors". On y lit (op. cit.: 196-197) que lors des funérailles collectives qui sont l'objet de ces rites, chaque défunt est représenté par un cauris et une natte et que ces "nattes sont repliées" (folded) de manière à former une "petite maison" (small house) qui sert d'abri au cauris correspondant". Pour sa part Ségurola (1963) écrit dans son Dictionnaire (s.v. $c$ (j) ${ }^{12}$, qu'au cours de ces cérémonies funèbres, qui ont lieu tous les quatre ou cinq ans, "on dresse une case en miniature pour chaque défunt"; pas plus que

11 Cf. supra, page 10.

${ }^{12}$ Ségurola (loc.cité.) traduit cís (mot que pour ma part je préfere écrire cŕys) par "un mort, un cadavre, un défunt", le terme renvoyant ainsi à divers aspects de la personne humaine (il ne s'emploie pas pour les animaux) telles qu'on la désigne, suivant les circonstances et le contexte, après son trépas. Il importe ici de faire apparaitre la notion latente qui fonde ces diverses acceptions du mort cŕy ou, pour formuler le problème dans les termes de la distinction préconisée par Benvéniste (1969, 1-12 et $4^{\circ}$ de couverture), de passer du niveau de la désignation à celui de la signification. Disons en bref, sans pour autant tomber dans le piège de la traduction, que "trépassant" offre en l'occurrence un accès commode à ce "niveau profond des significations" qui est celui qui nous intéresse. Par sa forme même, "trépassant" indique qu'il s'agit d'un processus en cours et "trépas", note Littré, "emporte dans son idée le passage d'une vie à une autre". Or l'analyse des divers usages du mot cío montre qu'après son trépas la personne humaine est vue comme passant par une suite d'états transitoires entre le moment où, morte (mekiukú), on se la représente comme hantant plus ou moins le monde des vivants et celui où, intégrée désormais au monde des morts (kútomє), elle est considérée comme faisant partie des défunts (kúvits, cf. ci-dessous la note 16 ). 
Herskovits il n'en indique le nom. Quant au mot ags, qu'il donne avec le sens de "grenier, magasin à provisions en vannerie", il le fait figurer à la suite, sans autre explication, dans l'expression $q u$ ag $f$ "faire un festin", locution extrêmement courante, on le verra bientôt, chez les Goun, puis dans le composé agł qut $\$, "festoyeur".

On retrouve ainsi chez les Fon, en premier lieu, à partir des textes de Herskovits et de Ségurola qui s'étayent l'un l'autre, la construction funéraire faite d'une natte repliée appelée agł par les Goun, en second lieu, à partir de Ségurola seulement, la notion de réjouissance (ou de "festin") qui est également celle de ce mot chez les Goun. La coïncidence a peu de chance d'être fortuite. Observons de surcroît que si dans le Ségurola le mot agð, "grenier" ou "festin", ne renvoie, contrairement à ce qui se passe chez les Goun, à rien de funéraire, il n'en désigne pas moins, comme en goun cette fois, une construction légère, faite en matière végétale ("en vannerie") et en cela tout à fait différente de la maison d'habitation aussi bien goun que fon, toujours massive et construite en "terre de barre".

Ici encore, cependant, une difficulté reste à résoudre. Elle tient à la présence, cette fois chez Herskovits comme chez Ségurola, du mot ag.sn pour désigner des réalités qui ne sont manifestement que d'autres aspects de ce qu'on vient de décrire et qu'on s'attendrait par conséquence à voir désigner du même mot, agł. Dans le Dictionnaire de Ségurola figure en effet, à côté de aģ (cf. qu agł et agłquţ) "festin", mais plus exactement, vient-on de voir, "réjouissances liées aux cérémonies funéraires de aģ", le mot agłn, traduit "réunion de la parenté pour l'offrande et l'exposition des pagnes destinés aux funérailles. Une partie sera enterrée, une partie ira aux fossoyeurs (...)". La cérémonie dont parle Ségurola est de toute évidence celle que décrit de son côté Herskovits, d'abord à la fin de ce qu'il appelle le "Partial burial" (Herskovits 1938 I: 364-65), puis, plus longuement, à divers moments du "Definitive burial". Disons, pour résumer très brièvement les choses dans la perspective qui nous intéresse ici, que cette cérémonie, ou plus exactement la suite des rituels dont elle se compose, est toute entière centrée autour de "temporary houses ... made of mats" (op. cit.: 364), dont on apprendra plus tard qu'elles (ou d'autres, mais 
n'entrons pas ici dans les détails) seront "détruites" et "brûlées" (ibid.: 393). Ces "maisons temporaires (...) faites de nattes", nous dit Herskovits, sont édifiées sur un espace dit agðn, situé à l'extérieur des habitations, face à leur entrée principale. Comme on voit, la seule différence dans l'emploi du mot agłn que font Herskovits et Ségurola consiste en ce que chez le premier il désigne le lieu où sont édifiées ces "maisons temporaires en natte" et chez le second l'assemblee qui s'y tient pour célébrer le rite funéraire qui leur est associé. Cas évident de métonymie ${ }^{13}$. On a vu que chez les Goun (voisins et proches cousins à tous égards, y compris par la langue, des Fon), la construction funéraire en natte, le rite de crémation et les réjouissances qui l'accompagnent sont appelés ag.s. On a également vu que chez les Fon ags (Ségurola, op. cit.) signifiait "festin" (i.e. réjouissances). Y aurait-il chez eux deux mots, ag§n et ag., dont le premier ne recouvrirait que certains aspects du rituel et le second les autres ? Compte tenu de ce que (fon) ags signifie également, on l'a dit, "grenier en vannerie" et par là construction proche de celle, funéraire, qui nous occupe, et de ce que (fon) agsn ne semble pas avoir d'autres significations que celle qui se rapporte au rite funéraire dont on vient de parler, cela paraît peu probable. S'agirait-il, dès lors, de deux formes différentes du même mot, résultat d'une alternance de voyelle où permutent nasale et orale ? Semblable permutation est extrêmement fréquente, d'un dialecte à l'autre, dans le groupe des langues dites gbe (ou tadoïdes, ou adjaévhé); le Worterbuch der Ewe-Sprache de Westermann (1954), en donne de nombreux exemples parmi lesquels, pour n'en citer que deux,

13 Le mot "agon", traduit "veillée", figure dans la description que donne B. Adoukonou de la cérémonie "cys do hun" pour servir de base à son étude sur " la mort dans la pensée adja-fon". Le contexte dans lequel apparait ce mot ne fait que renforcer mon hypothèse de la métonymie. Adoukonou (1979: 155) y parle en effet des jeunes gens que le "maître de cérémonic" envoie "détruire et brûler les cases de branchages" édifées peu avant pour la circonstance. Ces cases, avait-il indiqué auparavant (ibid.: 144), sont des "maisons symboliques" où l'on a couché des figurines de bois taillées pour "représenter" les "membres [du clan] décédés hors de l'enclos familial", objets de la cérémonie en question. Il est clair que ces "maisons symboliques" ne sont rien d'autre que les "maisons temporaires" décrites par Herskovits, et que les constructions funéraires dites ags chez les Goun. 
ga et gan, "métal", si et sin, "eau". Il y a, dans ces conditions, toutes les chances pour que cette seconde hypothèse soit la bonne et que, conséquence de ce qui a été dit plus haut de l'origine yorouba du mot, agł soit chez les Fon la forme première de ce vocable, agłn n'en étant alors que la forme dérivée, par nasalisation.

Que la forme a voyelle orale, agj, soit la plus ancienne, l'extension même du mot semble vouloir le confirmer. Elle seule est attestée, a-t-on vu, chez les Goun, sous-groupe le plus oriental du groupe oriental des langues adja-évhé. Elle l'est également chez les Adja, qui en sont le sous-groupe le plus occidental, ce qu'atteste sa présence dans le Dictionnaire Adja-Français (1969) de Harguindéguy - sous la seule forme d'un composé agłququ, "fête, réjouissance", il est vrai, mais il n'y a pas à s'en étonner, l'ouvrage étant, de l'aveu même de son auteur, très sommaire.

Quittons maintenant ces deux grands ensembles ethniques, adja (au sens très large du terme) et yorouba et venons-en, mais plus brièvement, à leurs voisins immédiats, à l'ouest des premiers, les Ashanti, et à l'est des seconds, les Edo de l'ancien royaume du Bénin (Bini).

Chez les Edo de Nigéria, nous apprend Bradbury (1957: 51) au sixième jour des rites mortuaires, une personne est choisie pour "représenter le défunt". Sous sa conduite, une procession se rend "en brousse", en un certain endroit où se trouve érigé, pour la circonstance, un bâti de perches recouvert d'une étoffe ("frame-work of sticks, covered with a cloth"). La personne en question fait mine de s'y asseoir. Finalement le bâti s'écroule ("the structure collapses") et ses éléments sont jetés au loin. Le rite est dit "Throwing away the sticks". Sans aller plus avant retenons ici que chez les Edo, comme chez les Goun et les Fon, le rituel funéraire comporte, à un moment donné, la construction d'une structure temporaire destinée à être presqu'aussitôt détruite.

A la Côte-d'Or, chez les Ashanti, rapporte Rattray (1927: 164166), on édifiait encore, au début du siècle, pour servir au cours du rituel funéraire, un abri sommaire ("a rough temporary hut") sous lequel on disposait, "le sixième jour apress la mort" un certain nombre d'ustensiles domestiques parmi lesquels une poterie neuve. On y ajoutait un peu plus tard, après avoir "enlevé les traverses" de la hutte 
et s'être adressé au défunt, une autre poterie, dont le couvercle avait été façonné à son image. Tout cela - je résume - était emporté le soir, après le coucher du soleil, en un lieu dit "au bois des ombres" ("to the thicket of ghosts"). Nouvelle variation, comme on voit, sur les deux thèmes complémentaires de la construction d'un abri temporaire, puis de sa destruction, et de son envoi vers l'autre monde, en même temps que les objets qui s'y trouvaient entreposés.

Ces quelques incursions, d'abord dans des territoires immédiatement voisins de celui qui nous occupe, ensuite dans deux autres plus éloignés, mais appartenant toujours, linguistiquement, au monde kwa, auront assez montré que ce que les Goun appellent, objets et rites, ags et qui se présente comme une coutume à bien des égards très particulière, ne leur est en réalité pas propre, mais existe également ailleurs, dans cette région de l'Afrique, sous des formes plus ou moins semblables. Réunion d'objets en rapport avec le défunt, érection d'une construction temporaire destinée à recevoir ou ces objets ou la représentation symbolique du mort, destruction, d'une manière ou d'une autre, de ces objets et/ou de cette construction, tels sont les éléments mis en auvre par ce rite, qui les combine, tous ou seulement certains d'entre eux, suivant des modalités variant avec les ethnies en cause, mais toujours en vue de favoriser, ici encore d'une manière ou d'une autre, l'intégration du défunt dans le monde des morts. Cette variété de combinaisons, dont une recherche plus systématique fournirait à coup sûr bien d'autres exemples, apparaît ainsi comme constituant ce que $\mathrm{Cl}$. Lévi-Strauss appelle un groupe de transformations. Que ces diverses constructions symboliques - le terme ayant cette fois les deux sens, concret et abstrait, d'abri temporaire et de représentations collectives le concernant - soient à considérer comme étant entre elles dans des rapports de transformation méritait d'être signale. Pour nous, le fait ainsi reconnu présente l'avantage d'autoriser, à l'intérieur du groupe bien entendu, certaines extrapolations d'un ensemble symbolique à un autre. Celui que constitue, chez les Goun, le rituel de ags pose, va-t-on voir, des problèmes d'interprétation que le recours à ces extrapolations aidera, précisément, à résoudre. Mais avant d'en venir aux interprétations, il nous faut d'abord achever l'examen des usages que l'on fait en goun de ags. 
En tant qu'objet, ag. se présente, a-t-on vu, chez les Goun, comme une chose vouée à être détruite. L'acte de détruire ag. s'énoncer de trois manières differrentes: on le brûle $(m \epsilon)$, on le verse $(k, 3 n)$ [dans le brasier], ou on le casse gba, ce qui donne lieu aux actions dites respectivement ags mime, ags kiksn et ag. gbigba. Le premier terme, "crémation de ags", est celui dont l'emploi est le plus fréquent. Il est aussi le seul susceptible de recevoir deux spécifications différentes permettant de distinguer deux rites: ag $\mathfrak{J}$ vivé mime, "crémation du agł coûteux" (vfvé: de vé, "être amer, douloureux, coûteux; cher dans tous les sens du mot") et ag’ mime qaxó ou gbó, "grande crémation de ag $\$$ ". Il s'agit dans le premier cas de la crémation qui a lieu soit le lendemain de l'inhumation, soit au terme de sept ou neuf jours durant lesquels on chante autour de la tombe les chants funebres dits sudehan ${ }^{14}$. Dans le second, de la grande crémation collective qui constitue l'un des moments forts de la deuxième phase des rites funéraires. C'est elle que l'on a en vue Iorsqu'on emploie, sans plus de précision, le terme de ags mime, "crémation de ags".

$A g 5$, en dehors de ses emplois avec $m \epsilon$, "brûler", gba, "casser", $k o n$, "verser", où il désigne le ou les objets que l'on brûle, casse ou met au feu, bref ce que l'on détruit rituellement, s'utilise avec wa, "faire" et $\phi u$, "manger, jouir de". Mi ná wa ags, "nous allons faire ag5", signifie sans plus de précision, que l'on va procéder à ce rite. $M t$ ná qu agł, "nous allons manger agł", signifie "nous allons goûter [les plaisirs de] ags", tout comme on dit, très couramment aussi, par exemple, "mí ná qu gbe, nous allons goûter [les plaisirs de] la vie". Dans cet emploi. ags ne renvoie évidemment plus aux choses que l'on brûle et autour desquelles s'opère ce rite de crémation, mais bien, par

14 Suqehan (interdit-enlever-chant) "chant de lever d'interdit". Décrivant comment s'exécutent ces chants funèbres, Akindélé et Aguessy (1953: 100) parlent du "pèlerinage du soundide". C'est sudide (interdiction-action d'enlever) "lever d'interdit" qu'il faut lire. Ce terme désigne différents types de rites de passage. En Haiti, le mot dessounin, qui désigne un rite funéraire (A. Métraux 1958: 217-218) n'est très vraisemblablement qu'une survivance du même terne, mais sous une autre forme dérivée sans doute de qesunie (enlève-interdit-pour lui/elle). 
évidente métonymie, au rite lui-même, ou plus exactement aux réjouissances qui lui succèdent et en constituent le véritable aboutissement.

Jusqu'aux années soixante et pour les personnes d'un certain âge en tout cas, le mot ne désignait strictement que les réjouissances faisant suite à la crémation de ag $\mathfrak{b}$, celles-là et aucune autre. Pour les générations suivantes, ou du moins celles qui, élevées en ville et coupées des anciennes traditions de lignage, ignorent jusqu'à l'existence de ces rites, le mot ag $\delta$ renvoie purement et simplement aux réjouissances et aux ripailles accompagnant toute fête collective. Que non seulement le sens premier du mot ait ainsi été perdu au profit de celui, dérivé, de réjouissances, mais que de plus le contexte funéraire de celles-ci en ait à son tour été évacué, tient de toute évidence à ce que le rite dit $a g s$ a fait une si grande place aux festivités qu'il en est devenu synonyme et a fini par en constituer le modele même. A l'occasion de $a g \mathfrak{y}$, des centaines de personnes, venues parfois de très loin, se réunissaient pendant plusieurs jours autour de la grande maison du lignage pour manger et boire à satiété, pour danser et chanter pendant des heures, chacun s'adonnant sans retenue aux joies de se trouver ou de se retrouver avec les siens.

Laissons maintenant ag 5 comme signifiant "réjouissances", ce qui n'est dans tous les cas, répétons-le, qu'un sens dérivé du terme, et revenons à ag $\$ comme objet ou collection d'objets destinés à être brûlés au cours des premières ou des deuxièmes funérailles. Brûlés pourquoi? Pour "empêcher l'esprit du propriétaire [i.e. du défunt]" de revenir les "visiter", nous disent Akindélé et Aguessy (1953: 109). Agগ, écrivent-ils par ailleurs (ibid:: 108), consiste en la "rupture des dernieres attaches naturelles avec le monde présent [ce qui est vrai pour le ag $\zeta$ des secondes funérailles, mais faux pour celui des premières]. Sans cette cérémonie, nous sentons trop intimement l'influence de nos défunts et ceci peut nuire à notre santé physique et mentale". Il s'agit donc avant tout, selon eux, d'empêcher les morts de venir troubler les vivants. Nul doute que ce soit bien, en gros, le but. Les choses ne sont cependant pas tout à fait aussi simples.

"On brûlera le ag $y$ coûteux ( $v i v e)$ pour celui qui vient de mourir", me disait un jour A. Dhènyon, le secrétaire du roi Gbèfa, "afin qu'il retrouve ses affaires et s'en serve dans l'au-delà ye ná me agj vívé ná 
me dée kú, ni dó mo nú éton lée zán olśn me)". Le ag̉ mime est un suqide ${ }^{15}$, un "rite [de passage]", ajouta-t-il, signifiant par là que si les vivants ont intérêt à respecter cette obligation c'est toutefois à l'intention du mort, premier concerné, qu'on l'exécute. Dans sa description, déjà citée, des funérailles des quatre derniers rois de PortoNovo qui eurent lieu en 1875, le R.P. Baudin (op. cit.: 615) écrivait:

"Les habitants de Porto-Novo [...] croient à une vie future; mais elle n'est, pour eux, que la reproduction ou la continuation de la vie présente. L'homme y est assujetti aux mêmes besoins, y éprouve les mêmes tristesses et les mêmes joies; et pour que les défunts ne soient pas, au-delà du tombeau, réduits à l'indigence, leurs amis de la terre vont brûler, hors de la ville, dans un bosquet mystérieux, tous les objets leur ayant appartenu ici bas. C'est ainsi qu'on leur expédie leurs nippes et leurs ustensiles de ménage. S'il s'agit d'un homme riche et puissant, on immole sur sa tombe ses femmes et ses esclaves."

Pour ce qui est du roi, certaines des victimes humaines immolees pour l'accompagner et le servir dans le monde des morts étaient jetées au bûcher au moment de la grande cérémonie. C'était notamment le cas, rapporte Baudin, de deux esclaves servant en quelque sorte de suppléants aux deux ministres Agbasagán et Sogán, respectivement chargés, du vivant du roi, de sa peau de panthère (agbasa) et de son cheval (so) et qui, dans les temps anciens étaient immolés en personne. Une jeune négresse destinée à "réjouir Leurs Majestés dans l'autre monde" était également jetée, plus ou moins vivante, dans le brasier où se consumaient les effets du roi défunt. Scènes horribles décrites avec complaisance par Baudin dans le but évident de dénoncer la barbarie des mœurs "fétichistes" et de justifier l'action missionnaire, mais scènes bien réelles, dont m'ont spontanément parlé divers PortoNoviens bien informés des choses du passé. Nonobstant leur cruauté, ces rites doivent donc être vus pour ce qu'ils sont et considérés comme découlant logiquement de la représentation générale de la mort que se

15 Suqiqe, cf. supra note 14. 
font les Goun; plus précisément de leurs idées concernant: 1) l'organisation d'un monde des morts conçue à l'image de celle des vivants; 2) les modalités du passage de ce monde-ci dans l'autre.

Toujours suspects de mauvais sentiments (jalousie, rancune) à l'égard des vivants, les morts sont spécialement soupçonnés d'intentions vengeresses pendant tout le temps qui sépare leur trépas de leur intégration définitive au monde des défunts, laquelle ne se réalise qu'au terme d'un long parcours dont ags marque une importante étape. La crémation des affaires du mort (agj mime) vise à munir celui-ci de ce qui va lui être nécessaire dans son existence - si l'on peut dire ainsi de "trépassant" (cíy), puis par la suite de défunt $\left(\right.$ kúvit $\left.3^{16}\right)$, et donc à désarmer son mécontentement éventuel, l'inégalité des sacrifices consentis à cette occasion illustrant simplement la règle "à chacun selon ses moyens".

Si le but de l'opération ne fait pas de doute, la logique des moyens mis en cuvre apparaît moins clairement, aux yeux de l'observateur étranger tout au moins. Par quelle grâce mystérieuse ces objets réduits en cendre recouvrent-ils leur fonction utilitaire dans l'autre monde? Par quel jeu de transsubstantiation y retrouvent-ils une nouvelle réalité ? Poser la question, c'est s'interroger sur la conception qu'ont les Goun de l'invisible et plus généralement sur les fondements de la pensée symbolique à l'origine, chez eux comme chez tant de peuples, de ce type de représentation. Comme chacun sait, chez les Grecs les dieux se repaissaient de la fumée et du fumet des viandes rôties en leur honneur; autre aspect, à l'évidence de la crémation. Celle-ci serait-elle liée, ici et là, en partie au moins, à des idées

${ }^{16}$ Kúvils (mort-calmer - n.a.), "celui/celle qui calme la mort". Les küvits sont les défunts consacrés comme tels, ayant leurs autels commémoratifs, asánnyi, où s'effectuent les libations propitiatoires dont ils sont l'objet. Vi, "calmer" entre dans la composition d'un autre terme, novits (mère-calmer - n.a.) "celui/celle qui calme la mère", lequel désigne le représentant ou la représentante d'un certain lignage investi du pouvoir d'intervenir pour calmer les querelles et régler les conflits survenant dans un autre lignage. Que le mot kúvits (variantes kúbits, mais aussi kútíts, pour laquelle je n'ai pas d'explication) s'analyse comme il vient d'être dit me semble peu contestable. Les représentations qui lui correspondent n'en restent pas moins à élucider. 
concernant les transformations de la matière opérées par le feu ? Bornons-nous à enregistrer les faits tels qu'ils se présentent chez les Goun. Mais signalons cette fois encore qu'ils ne sont ni aussi simples ni surtout aussi univoques qu'on pourrait le penser.

Deux choses sont à considérer dans cette crémation: la finalité qu'on lui assigne d'une part, de l'autre la manière qu'on a d'y procéder. Or la seconde contredit assez largement la première. Le comportement de ceux-là mêmes qui participent, tous ensemble, à la crémation fait douter en effet que la sollicitude pour le mort, prétendument seule raison d'être de l'action qu'ils sont en train d'accomplir, soit bien l'unique sentiment qui les anime. Cette remarque se fonde en grande partie sur ce qu'il me fut donné de voir au cours de cette cérémonie de agł vívé mime, "crémation du agł coûteux", dont j’ai déjà parlé plus haut et qui eut lieu le 2 janvier 1959 chez des paysans, dans un hameau des environs de Porto-Novo. Il nous faut maintenant entrer un peu dans le détail.

Une femme était morte une quinzaine de jours auparavant. Sous la direction de son frère aîné, ses proches s'apprêtaient à chanter pour la septieme et dernière fois la série des sept sudehan ${ }^{17}$, "chants funèbres" de rigueur en pareil cas, mais cette fois à les chanter dehors, sur la place centrale du hameau et non plus, comme les jours précédents, autour du tombeau qui avait été creusé pour elle à l'intérieur même de sa chaumière. Par terre était posée, sur une bouse de vache séchée, une petite poterie contenant de l'eau dont chanteurs et chanteuses - six à huit personnes en tout - commencèrent par boire chacun une gorgée, en se servant, pour puiser le liquide, d'une cuillère faite d'un fragment de calebasse. Précédé par le frère aîné, qui faisait office de soliste, le petit groupe, disposé en file, se mit à tourner lentement en cercle autour de la poterie dans le sens dextrogyre, et exécutèrent ainsi, à la suite, six de ces chants funèbres, entonnés d'abord par le chanteur soliste. Pour le septième et dernier de la série,

17 Suqehan, cf. supra note 14. 
le cercle s'immobilisa, chanteurs et chanteuses faisant alors face a la poterie, posée à leurs pieds, puis se baissant progressivement vers elle à mesure qu'ils faisaient entendre le chant dont voici les paroles:

"(chanté)

Solo a bo ji aji qé to gbe

Chœur a ná mo to xwé ogidi ogodo, ogidi ogodo

Solo a bo $x$. me dé to gbe

Chneur a ná mo to xwé ogidi ogodo, ogidi ogodo

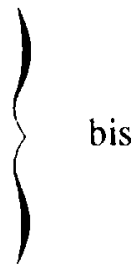

(au bis, cette dernière phrase est chantée en tout six fois, diminuendo)

(parlé)

(chanté)

Solo Si tu as fait un enfant dans la vie [i.e. ici bas]

Chœur tu le [re]trouveras à la maison [i.e. dans l'au-delà] écrase, écrase, écrase, écrase

Solo Si tu as acheté quelqu'un [i.e. un esclave] dans la vie

Chour tu le [re]trouveras a la maison

(parle) écrase, écrase, écrase, écrase (six fois)

un.. deux.. trois.. quatre.. cinq..six.. sept."

A la fin du chant, les participants, penchés en avant, étaient si serrés les uns contre les autres, que les têtes se touchaient comme dans une mêlée de rugby. A "sept" il y eu comme une soudaine bousculade, chacun se précipitant pour casser, du poing ou du pied, la poterie qui fut en un instant réduite en morceaux. Quelques rires fusèrent. Pour ma part, j'eus le sentiment que dans la hâte et dans la brutalité mise par le groupe tout entier a détruire cet inoffensif objet entrait une certaine part de joie mauvaise. La traduction des paroles du chant, quelques jours plus tard, vint confirmer mon impression. Comment ne pas voir de la dérision non seulement dans ce que l'on venait de chanter au mort (pour le consoler apparemment), mais encore dans les paroles choisies pour le faire: "Si tu avais un enfant, un esclave dans la vie (!), tu les retrouveras à la maison (!)" ? Comment ne pas voir 
de la méchanceté dans cet acharnement à détruire, immédiatement après ce discours et comme pour bien montrer la réalité des choses, cet ustensile de ménage ayant selon toute vraisemblance appartenu à la morte. Comment ne pas être confirmé dans cette interprétation par les rites furtifs qui suivirent? La suite du rituel ne fit qu'ajouter de nouvelles raisons de croire à la profonde ambiguïté de ce comportement.

L'exécution de la suite des sept chants funèbres s'étant terminée comme il vient d'être dit, une fillette ramassa les morceaux de la poterie brisée et les rangea en tas sur la bouse de vache qui venait de lui servir, en quelque sorte de soucoupe. [La coutume veut, ai-je appris plus tard, qu'une fois cette poterie cassée, un "oncle" (taffe) ou une "tante" (tányi) ${ }^{18}$ demande: "Où sont les enfants (vívú lée) ?" C’est alors que la fillette - vierge, fut-il précisé - celle-là même qui a préalablement mis en place la bouse de vache, entreprend d'y remettre, de la main gauche, ce qui reste du pot. D'autres enfants viennent l'y aider. Je n'eus pas le temps de le noter, mais il y a toutes les raisons de penser que c'est effectivement ce qui eut lieu ce jour-là.l Après quoi, des grains de maïs furent disposés tout autour de ces débris et quelques très petites fleurs (?) de couleur violette éparpillées de-ci delà. Cela fait, on posa par-dessus le tout, comme s'il s'agissait de l'abriter, la natte repliée en forme de tente (ags) ou de toit dont on a parlé tout à l'heure.

Munis de ce qui a été dit précédemment de aģ, nous pouvons maintenant nous interroger sur cette curieuse conjonction de natte repliée, de bouse de vache et de poterie brisée. Que signifie-t-elle ?

Que la natte repliée soit un simulacre d'abri temporaire - simulacre, parce bien trop petit pour servir à un humain - c'est ce qu'indiquent non seulement l'évidence de sa forme mais encore les termes utilisés pour la désigner: (yor.) aģ, d'abord, bien sûr, mais aussi "case" (Baudin), "case en miniature" (Ségurola), "small house" (Herskovits). Cela étant, abri ou maison de ou pour qui ? La destination de cette construction temporaire, vouée à être brûlée avec les affaires du mort, donne évidemment à penser que c'est de la maison

${ }^{18}$ Táfe, tányi, "oncle", "tante", paternels. 
du mort, ou du moins de sa figuration symbolique qu'il s'agit. Les choses - et s'il est le roi, les gens - qu'on lui expédie dans l'autre monde en les brûlant sont destinées a le pourvoir de ce dont il aura besoin dans son existence de "trépassant" (ctyo) d'abord, puis de défunt. Quoi de plus nécessaire qu'une maison? Les paroles mêmes du dernier chant qu'on adresse au mort avant de procéder à la crémation de son ags ne disent-elles pas, comme on vient de le voir:

"(Ce que tu avais dans la vie)

"Tu le retrouveras à la maison ?"

Il y a là, on en conviendra, de quoi conforter l'hypothèse suivant laquelle ag $\zeta$ serait en définitive la figuration symbolique de la maison du mort, ou plus exactement de son abri temporaire. Temporaire parce qu'en yorouba, a-t-on dit, le mot ag 5 désigne une construction provisoire, mais peut-être aussi parce que le caractère provisoire de cette construction traduit, précisément, l'état du mort pour lequel elle est érigée, le mort étant vu (si mon interprétation du mot cíys est exacte; sur ce point voir note 12) comme occupant temporairement une situation intermédiaire entre celle des vivants et celle des défunts.

Passons maintenant à la bouse de vache. En pays goun, il en est fait un usage très particulier: diluée dans un peu d'eau, on en enduit le sol de la maison et le bas des murs, en vue de l'entretien et de l'embellissement des lieux. A Honme, les plus jeunes femmes du roi procédaient régulièrement à cette opération. "On fait cela dès qu'il y a trop de poussière", me dit un jour le roi Gbèfa, qui ajouta, pour mieux me faire comprendre: "C'est notre ciment". L'abbé Bouche (1875: 290), en visite au palais, avait déjà observé le fait du temps du roi Mikpon, mais bien avant lui le Captain J. Adams (1823: 81) avait signalé cette "singular custom" comme étant d'usage courant à Porto-Novo, observation faite a nouveau soixante-dix ans plus tard par Foa (1895: 180) et toujours valable, vient-on de voir, à la mi-temps du siècle suivant. La bouse de vache apparaît ainsi comme étroitement liée au bon état des murs et du sol de la maison. Cela étant, on serait tenté de penser que dans le cas du rituel funéraire qui nous occupe la fonction symbolique de la bouse de vache est de signifier, par sa seule présence à terre, que l'abri sous lequel elle se trouve est une habitation. La 
conjonction de ces deux éléments, natte repliée et bouse de vache, exprimerait ainsi, par homologie, le caractère essentiel de la maison vue comme l'association d'une toiture et d'un sol aménagé. Faute d'informations plus précises, telle est, semble-t-il, la lecture la plus cohérente que l'on puisse offrir de cette construction (aux deux sens du terme) symbolique.

Venons-en maintenant à la poterie brisée. Chez les Goun, comme souvent ailleurs en Afrique, poterie et eau à boire sont par excellence choses féminines. Or il s'agit ici des funérailles d'une femme. Ainsi s'expliquerait peut-être la place importante dévolue à cette poterie dans le rituel. Mais qu'il s'agisse ou non d'une particularité des funérailles de femme importe à vrai dire assez peu ici. C'est le traitement subi par l'objet, le fait d'être mis en morceaux qui nous intéresse. Briser une poterie à un moment donné des funérailles est en effet, chez les Goun, une pratique coutumière ${ }^{19}$. Chez les Dravonou, sous-groupe goun de Porto-Novo (Marie-Josée Pineau-Jamous, com. pers.), les débris de la poterie (zén) ainsi cassée au cours de la cérémonie de agł, sont arrosés du sang d'un porc (han) sacrifié pour la circonstance et appelé pour cette raison agł zén kijá han, "porc de la poterie brisée de agł"; dénomination qui, par son existence même montre assez que briser une poterie constitue une action rituelle bien définie, répertoriée et habituelle. $K(j a ́$, lit-on dans Ségurola, signifie "briser avec violence". Nous nous demandions tout à l'heure, "pourquoi brûler agł ?" La même question se pose maintenant; pourquoi casser, au cours de ce rite, une poterie? A Abomey, chez les Fon, écrit Suzanne Preston Blier (sous presse): "Si quelqu'un veut envoyer une poterie aux kulito [en goun lisez kúvity] (les morts), il doit d'abord la casser". Le bris de la poterie apparaît ainsi comme dicté par la même idée que la crémation des autres affaires du mort: pour rejoindre celui-ci dans l'au-delà, l'objet

${ }^{19}$ Rite que l'on retrouve, comme on sait, en Haïti sous le nom de "casser-canari" (A. Métraux 1958: 224-226). 
quel qu'il soit doit être au préalable, d'une manière ou d'une autre, détruit ${ }^{20}$.

Ainsi doivent très certainement s'interpréter, par conséquent, aussi bien la poterie brisée que le fossoyeur fon jette dans la tombe avant de la combler, pratique décrite sans explication par Herskovits (1978: I, 357), que les "assiettes de valeur cassées dans le feu", dont parle, a-t-on vu, Kiti chez les Goun. Peut-être faut-il interpréter également de cette manière le "Tambour du roi brisé en morceaux" que déposent les Yorouba de Ifé, sur le tombeau de leur souverain (Oni) défunt (W. Price 1939, cité par M. Palau-Marti 1964: 23) ou, chez les Ashanti, la poterie contenant trois cailloux que le veuf ou la veuve brise en la laissant tomber à terre au cours des funérailles, à leur arrivée au cimetière (Rattray 1927: 161). Nous sommes encore là, en effet, dans le périmètre de ce groupe de transformations dont on a reconnu tout à l'heure l'existence. Mais briser des objets ayant appartenu au mort est une pratique funéraire très répandue et qui le déborde, en conséquence, largement. Pour n'en citer que trois exemples en Afrique de l'Ouest, au Sénégal, chez les Bedik, "on casse la poterie à eau d'une femme sur sa tombe" (M.-P. Ferry 1991: 390), tandis que chez les Konyagi, leurs voisins, on casse, pour les hommes, leurs $\operatorname{arcs}^{21}$ (ibid.: 391). Au Mali, chez les Dogon, c'est le manche de la

20 Dans la description qu'a laissé Labarthe (1803: 123-125) de l'enterrement du roi à Abomey, parlant des veuves du défunt que l'on "voit se presser à qui descendra la première dans le tombeau", il écrit: "Un usage qui ajoute à la barbarie de ces coutumes, c'est qu'auparavant ces femmes doivent avoir les jambes cassées, ce qui s'exécute à coups de masse". Il s'agissait là, lit-on plus haut, de "précaution", certaines victimes (des hommes cette fois) "qui n'avaient pas eu les jambes brisées" ayant réussi, naguère, à s'enfuir. On est tenté de se demander s'il ne s'agirait pas, en fait, d'une application - particulièrement dramatique - de ce principe voulant que ce qui doit aller rejoindre le défunt soit auparavant "brisé".

21 Chez les Kasena du Burkina-Faso (D. Liberski 1989: ix-x), l'are du défunt est d'abord "brisé", puis au cours d'une séquence ultérieure du rituel "une reproduction miniature du carquois du mort sera détruite par le feu". Chez les Tamberma du Togo, l'arc du mort est "renversé" et mis auprès du corps que veillent les femmes; on pose l'arc "tête en bas" sur le sol afin de le débander, nous apprend M. Smadja (1991: 70) dans une étude particulièrement riche en informations sur le thème de la destruction des "affaires du mort". 
houe dont se servait le défunt qui est cassé et jeté dans la brousse au moment de ses funérailles (Leiris 1948: 221). Ces bris rituels d'objets ne correspondent pas nécessairement, chez ces dernières ethnies, aux mêmes représentations symboliques que celles de Goun, des Yorouba ou des Ashanti. Les unes et les autres n'en sont sans doute pas moins entre elles, au sein d'un groupe plus vaste et se définissant différemment par conséquent, dans des relations de transformation. Il n'est pas de notre propos de les examiner, mais il est hors de doute que c'est seulement en les replaçant dans cet ensemble que l'on pourra prétendre à dégager la signification profonde de ces destructions rituelles d'objets ayant appartenu au mort. Pour nous, ici, contentons-nous de retenir que le bris d'une poterie, qui marque un des épisodes des cérémonies de $a g \hat{\jmath}$, répond, tout comme la crémation d'autres affaires du défunt, au devoir de le munir des objets nécessaires à son existence quotidienne dans l'au-delà.

Revenons maintenant à notre agj paysan du 2 janvier 1959. La natte repliée ayant été installée, comme on l'a vu, au-dessus des débris de poterie (notre photo), ceux-ci furent arrosés du sang d'une poule sacrifiée. Un porc fut égorgé. Diverses actions, que je n'eus pas le temps d'observer, se déroulèrent alors rapidement, après quoi l'on se forma en cortège pour aller procéder, hors du village, au rite du ags vivé mime, la "crémation du ag $\zeta$ coûteux". Outre la natte et les débris de poterie dont on vient de parler, le agł, réparti entre différentes personnes du groupe se composait d'un petit nombre d'objets ayant appartenu à la morte: lit de bambou plus ou moins démantibulé, ustensiles de cuisine, calebasse, bidons hors d'usage, morceaux d'étoffe. A la sortie du hameau avait été édifié un léger portique en tiges de bambou. On y fit halte un instant, pour permettre aux jeunes gens de la troupe de se munir de quelques bâtons avec lesquels rythmer, en les entrechoquant, les chants qui allaient maintenant accompagner la marche du cortege vers sa destination. Ces suqehan, ces "chants funèbres" étaient, m'a-t-on dit, de ceux qui sont particulièrement destinés à cet épisode de la crémation. Parvenus, après une très courte marche, au agj to, au "lieu de agł", situé en bordure du sentier et ressemblant, pour tout dire, à un dépôt d'ordure, on y jeta pêle-mêle le "bagage de agł", agł gban, qu'on arrosa d'un peu d'essence pour 
y mettre le feu. Si mes souvenirs sont exacts, on resta là un moment, à chanter et à danser sur place, en battant des mains et en entrechoquant les bâtons, cependant que certains buvant à la bouteille quelques gorgées d'alcool local (sodabi), le recrachaient en pluie sur les flammes pour les aviver. On jeta également au feu quelques piæces de monnaie. Toujours dansant et toujours chantant ces suqehan funèbres, on s'en retourna bientôt au village. Arrivée au portique de bambou, la petite troupe fit halte. Une cuvette remplie d'eau avait été disposée par terre. Chacun à son tour y trempa un bouquet de feuilles pour s'en asperger, - rite de purification, à l'évidence, homologue de celui que décrivent Akindélé et Aguessy (1953: 107) sous le nom de houékpikplo [xwé kpikpl's], "purification de la maison", ou de celui que pratiquent certains lignages porto-noviens dont les membres, au retour d'un ags mime, vont se tremper les pieds dans la lagune avant de rentrer chez eux. On laissa là les bâtons dont on s'était servi pour rythmer, de leur entrechoc, les chants funebres. La rentrée au village s'accompagna de chants tout à fait différents, manifestement très gais. "Tout le mauvais est resté là-bas" me cria, hilare, le frère aîné de la morte, "maintenant c'est le bon que nous allons trouver". Ainsi, de son propre aveu, les affaires $(a g \mathfrak{g})$ que l'on était allé brûler en brousse pour que la défunte "les retrouve et s'en serve dans l'au-delà", comme disait le secrétaire, "c'etait du mauvais!" Et ce mauvais, je ne le lui faisais pas dire, on venait, pour parler clairement, de s'en débarrasser. Eloigner la morte et avec elle la mort, et puis, cela fait, donner libre cours à la joie de se retrouver tous ensemble, bien vivants, c'est en réalité de cela qu'il s'agissait. La tristesse et le deuil qui s'exprimaient peu de temps avant à travers les suđehan, les "chants funebres", n'en avaient pas été, pour autant, moins sincères. La mort, chantait-on, est cruelle. Il faut donc savoir, cruellement au besoin, s'en défendre.

Mais, dira-t-on, ce double aspect des cérémonies de ags, acceptation et tristesse de la mort en même temps que revanche et joie de la vie, n'est-il pas à quelque degré, celui de toute cérémonie funéraire ? Sans entrer dans le débat, versons au dossier deux remarques. La première est que chez les Goun le contraste entre ces deux aspects de la cérémonie est particulièrement marqué. Ou tout au moins que dans certains cas, comme celui que l'on vient de relater, il arrive que, le 
moment venu, la joie s'exprime sans retenue et vienne brutalement mettre un terme aux manifestations de tristesse - très contenues, au demeurant - qui précèdent. Hâtons-nous de dire, cependant, que tout dépend, dans une certaine mesure au moins, de la situation et qu'en l'occurrence, s'agissant d'une personne déjà un peu âgée et dont la mort était plus ou moins attendue, elle n'avait pas ce caractère dramatique qu'entraîne la mort subite de quelqu'un de jeune. Il est à penser que dans ce dernier cas le contraste entre l'expression des deux sentiments eût été inversé et que la douleur l'eût alors emporté de beaucoup sur la joie. Mais notons-le, car c'est ce qui importe, celle-ci n'en aurait pas été oubliée pour autant. Son tour venu, elle aurait eu, bon gré mal gré, sous la contrainte du rite, à s'exprimer.

Observons en second lieu qu'en tant que consistant essentiellement à expédier au mort, pour qu'il puisse s'en servir dans l'au-delà, des objets préalablement détruits (brûlés ou cassés), le rituel de ag $\Im$ apparaît comme étant fondamentalement ambigu. Décrivant un peu plus haut le comportement de ceux qui participèrent à la crémation des affaires de la défunte au cours du ags mime du 2 janvier 1959, j'ai dit l'impression - subjective, sans doute - d'acharnement destructeur, de joie mauvaise, de dérision qui me paraissait s'en dégager. On aurait cru, pour tout dire, qu'on en voulait à la morte d'être morte et qu'on l'en punissait. Ou encore qu'on l'en moquait. D'autres données sembleraient confirmer l'existence d'une composante en quelque sorte perverse de ces conduites rituelles à l'égard de la mort. Perverse parce que consistant à tourner celle-ci en dérision, et atteignant en fin de compte ainsi tout autant les vivants que les morts.

Dans la description que fait l'abbé Kiti (1968: 17) d'un épisode du rituel funéraire (distinct, à vrai dire de ags, mais venant immédiatement avant lui), on lit qu'avant d'aller enterrer définitivement les jarres contenant les crânes des défunts "les assistants se précipitent sur les ornements dont les pots sont couverts et commencent à se les disputer. Tout en défendant les siens, chacun cherche à voler ceux des autres". Kiti n'en dit rien, mais on est évidemment en présence, ici, d'une parodie, autrement dit d'une conduite de dérision et par là même d'une provocation à l'égard du respect dû aux morts. Pour sa part, Herskovits (1938: I, 376 sq.) parle également, chez les Fon, d'une 
parodie de lutte ("mock fight") qui prend place au moment où l'on procède à la destruction du Légba ("ange gardien", traduit-il à tort; en fait effigie d'un des aspects de la personne) du défunt. Mais plus provocante encore apparaît une autre pratique rituelle qu'il décrit peu après (ibid.: 389) et qui consiste à se livrer à la fornication sur le lieu même où vient d'être inhumé définitivement le cadavre.

On sait assez combien l'interprétation d'actions symboliques de ce type requiert de prudence. Proposer de voir dans les diverses pratiques considerées, depuis la destruction, sous différentes formes, des objets expédiés au mort jusqu'à la fornication sur sa tombe, des rituels également empreints d'une certaine volonté de dérision, est assurément hasardeux. Par ailleurs les réunir, comme on vient de le faire, pour en signaler l'existence, c'est déjà, de toute façon, les interpréter. Il m'a semblé que quels que soient les risques, tout valait mieux que d'ignorer les faits et de les passer sous silence. Ajoutons que cette volonté de tourner la mort en dérision - à supposer qu'elle existe effectivement reste à expliquer, c'est-à-dire à replacer dans le tableau général des représentations de la mort que se font les Goun. Resterait aussi a savoir de quel crédit bénéficient réellement ces croyances ${ }^{22}$.

Venons-en pour finir au composé de agł, agł quhún (agł - goûter [le plaisir de] - tambour), litt. "tambour-du-goûter-de-ag 3 ", terme qui pourrait se traduire par "musique de réjouissance". Le mot ag. s'emploie, rappelons-le, pour désigner deux rituels bien distincts: le premier ag $\delta$ vívé mime, "crémation du ag $\delta$ coûteux", suivant de plus ou moins près l'inhumation, le second, ags mimé qaxó, "grande crémation de

22 "... although the Yoruba generally believe in posthumous existence, yet they often express great doubts about its certainty and the replication of life-type pursuits and pleasures in ghostland", écrit Isola Olomola (1988: 116) dans une étude où il est très souvent question de ces (yor.) Egúngün, "Revenants", que les Fon et les Goun désignent souvent du nom de kúvits (ou kúbits) plusieurs fois mentionné ici.

Certain jour de 1987 , m'entretenant de divers sujets avec l'awatagan Tchèdji qui avait été l'awatagan - i.e. le ministre chargé d'annoncer la mort au roi - de feu le roi Gbèfa et qui avait alors quatre-vingt sept ans, je fus amené à lui demander si, comme on le croit en général, au pays de la mort (kútome) on fait tout à l'envers, on passe les portes à reculons, on mange de la main gauche. "C'est ce qu'on dit", me répondit-il très flegmatiquement, "mais pour ma part je ne sais pas vraiment comment se passent les choses au pays de la mort, car je n'y suis jamais allé". 
ag $\zeta^{\prime}$, se célébrant beaucoup plus tard, après l'exhumation du ou des crânes. C'est exclusivement a l'occasion de cette "grande crémation", qui donne lieu à de très importantes festivités collectives, qu'a lieu cet agłquhún, cette "musique de réjouissance". Mais à vrai dire ce n'est là que le sens second du terme qui désigne, d'abord, un groupe de musiciens - instrumentistes et chanteurs - réunis autour d'un tambour (hún) et constituant un ensemble de semi-professionnels dont on loue les services pour la circonstance. Il existe en pays goun divers types d'ensembles de ce genre, chacun se caractérisant par ses instruments de musique et par son répertoire; les principaux sont ceux dits akshún, sát’, húngán et ketehún. Agł quhún, qui les recouvre tous et convient aussi bien à l'un qu'à l'autre, est donc un terme générique négligeant leurs spécificités organologiques et musicales et ne retenant que leur fonction, celle de fournir la musique destinée à permettre de, littéralement, "manger" ags.

Gilbert Rouget

Références biblicgraphiques

Abraham, M. C.

1958 Dictionary of Modern Yoruba, London, University of London Press.

Adams, Captain John

1823 Remarks on the Country Extending from Cape Palmas to the River Congo, London, Whittaker.

Adoukonou, Barthélemy

1979 "Pour une problématique anthropologique et religieuse de la mort dans la pensée adja-fon" in Y. K. Bamunoba \& B. Adoukonou, 1979, pp. 116-330. Cf. Bamunoba. 
Akindélé, A. \& Aguessy, C.

1953 Contribution à l'étude de l'histoire de l'ancien royaume de PortoNovo (Mémoires de l'Institut Français d'Afrique Noire, $n^{\circ} 25$ ), Dakar, IFAN.

Anonyme

1937 A Dictionary of the Yoruba Language, London, Oxford University Press.

Bamunoba, Y. K. \& Adoukonou, B.

1979 La mort dans la vie africaine, Paris, Présence africaine et Unesco.

Baudin, R. P.

1875 "Funérailles des quatre derniers rois de Porto-Novo", Les Missions Catholiques, Tome VII: 614-616 et 627-628, Lyon.

Benveniste, Emile

1969 Le vocabulaire des institutions indo-européennes, 2 vol., Paris, Les Editions de minuit.

Blier, Suzanne Preston

1992 The Art of Assemblage: Aesthetic Expression and Social Experience in Danhomè (sous presse).

Bouche, Abbé Pierre

1885 La côte des Esclaves et le Dahomey, Paris, E. Plon, Nourrit et Cie.

Bradbury, R. E.

1957 The Benin Kingdom (...), (Ethnographic Survey of Africa, Western Africa, Part XIII), London, International African Institute.

Ellis, A. B.

1894 The Yoruba-speaking Peoples of the Slave Coast of West Africa, London, Chapman and Hall.

Ferry, Marie-Paul

1991 Thesaurus Tenda. Dictionnaire ethnolinguistique de langues sénégalo-guinéennes (3 vol.), Paris, SELAF, Peeters.

Foa, Edouard

1895 Le Dahomey, Paris, A. Hennuyer. 
Harguindéguy, R.

1969 Premiers Eléments pour un Dictionnaire Adja-Français (2 vol.), Lyon, Afrique et Langages.

Herskovits, Melville J.

1938 Dahomey. An Ancient West African Kingdom (2 vol.), Evanston, Northwestern University Press.

Kiti, R. P. Gabriel

1968 "Rites funéraires usités chez les Alladanou et diverses tribus de race goun ou aizo habitant la banlieue de Porto-Novo", Etudes Dahoméennes, N.S., $\mathrm{n}^{\circ} 11$, Porto-Novo. [Repris de Revue des Missions catholiques], Lyon 1929 et 1930.

Labarthe, P.

1803 Voyage à la Côte de Guinée, Paris, Debray.

Leiris, Michel

1948 La langue secrète des Dogons de Sanga, Paris, Institut d'ethnologie.

Liberski, Danouta

1989 Présentation de Le deuil et ses rites, Systèmes de pensée en Afrique noire, IX, vii-xiii.

Maupoil, Bernard

1943 La Géomancie à l'ancienne Côte des Esclaves, Paris, Institut d'ethnologie.

Métraux, Alfred

1958 Le Vaudou haïtien, Paris, Gallimard.

Olomola, Isola

1988 "Contradictions in Yoruba folk beliefs concerning post-life existence: the Ado example", Journal des Africanistes, tome 58-1: 107-118.

Palau-Marty, Montserrat

1964 Le roi au Bénin, Paris, Berger-Levrault.

Rattray, R. S.

1927 Religion and Art in Ashanti, London, Oxford University Press. 
Rouget, Gilbert

1972 "La nasalisation des voyelles et le système des consonnes en gun" in J. Thomas et L. Bernot éd., Langues et techniques. Nature et société. (Hommage à A. Haudricourt), 1972: 209-219.

1974 "Tons, intonation, accentuation: problèmes d'identification", Les langues sans tradition écrite (Actes du Colloque International du C.N.R.S., Nice, 1971), 1974: 217-240.

1991 "Musique et royauté en Afrique", CNRS-INFO, Numéro spécial "La musicologie au CNRS", pp. 9-10, Paris, CNRS.

Ségurola, R. P. B.

1963 Dictionnaire fon-français (2 vol.), Cotonou, Procure de l'Archidiocèse.

Smadja, Myriam

1991 "Les affaires du mort (Tamberma, Nord-Togo)", Le deuil et ses rites II, Systèmes de Pensée en Afrique Noire, XI, 57-89.

Westermann, Diedrich

1954 Wörterbuch der Ewe-Sprache, Berlin, Akademie-Verlag. 